\title{
ARE QUANTUM FIELDS CELLULAR AUTOMATA?
}

\author{
Karl SVOZIL \\ Atominstitut der Österreichischen Universitäten, Schüttelstrasse 115, A-1020 Vienna, Austria \\ and Institute for Theoretical Physics, Technische Universität Wien, Karlsplatz 13/136, A-1040 Vienna, Austria
}

Received 7 May 1986; revised manuscript received 14 September 1986; accepted for publication 1 October 1986

\begin{abstract}
Tesselation of the quantum field and local connectivity of the cellular automaton type is investigated. A connection between this class of models and lattice field theory is established. Nonlocal models are briefly discussed.
\end{abstract}

1. The introduction of cellular automata in theoretical physics [1] initiated several ingenious speculations [2-6] concerning their applicability to field theory, culminating in a sort of "atomization" of a continuous field function $\varphi(t, \boldsymbol{x})$ into discrete lattice states

$$
\varphi(t, \boldsymbol{x}) \rightarrow \varphi_{n, \boldsymbol{n}}^{(i)}
$$

of locally (usually nearest neighbor) connected array elements evolving in time steps. This procedure is often called tesselation; $i$ as well as $n$ and $\boldsymbol{n}$ are discrete time and space parameters.

2. The intention has been not just to simulate a continuous field phenomenology, but rather to consider field atomization as factual. According to this approach, the perception of a seemingly continuous field is caused by insufficient experimental space and time resolution. In this picture, the macroscopic laws are the yield of elementary microprograms on the cellular automaton level. Hence, given a sufficiently large number of locally connected array processors $\left(10^{100}\right.$ per cubic meter or so [5]), it would be possible to reproduce field theory exactly by discretizing the states $\varphi^{(i)}$ as well as the coordinates $n, n$ of the continuous field $\varphi(t, x)$. The amount of information per unit volume of space-time would be finite. The macroscopic time evolution would consequently be the result of some local microscopic cellular automaton rule $\tau$ symbolized by

$$
\varphi_{n+1, n}=\tau\left(\varphi_{n,\{n\}}\right),
$$

where $\varphi_{n,\{n\}}$ characterizes an $n$-dimensional neighborhood (naively three-dimensional), including the kernel $\varphi_{n, n}$ itself. As noted earlier, the field function $\varphi$ takes on a countable (or even finite) number $N=\operatorname{card}\left(\left\{\varphi^{(i)}\right\}\right.$ ) of states.

3. The following observation concerning the topology of tesselations will be useful: when the $n$-dimensional cells (into which space-time is divided) are contracted to singular points such that their connections (topologies) are kept, a dual lattice is produced (see fig. 1). From a topological point of view, the lattice representation is equivalent to a cellular automaton. In that way, results from lattice theory can be readily applied to cellular automata theory and vice versa.

4. It will be argued, that field theory cannot be discretized in the way envisaged above. However, one way to surmount difficulties would be to give up the condition of locality. The major support for the argument comes from a no-go theorem [7-9], stating that for a very general class of fermion lattice theories under "mild 


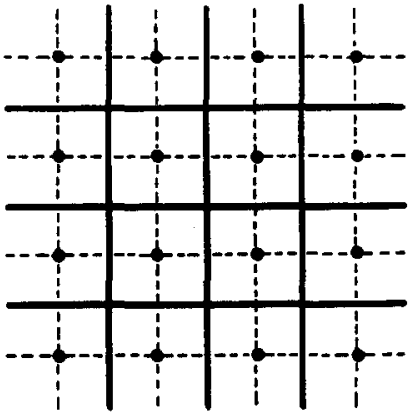

Fig. 1 .

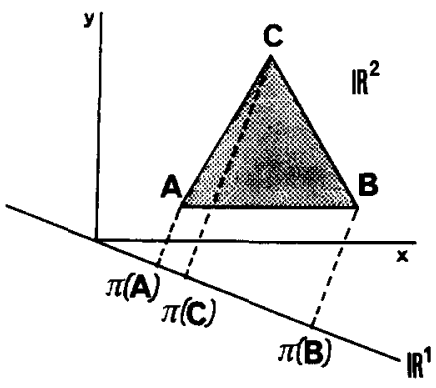

Fig. 2.

assumptions" it is not possible to construct unambiguous dispersion relations, such that for vanishing energy there is only one allowed value of the momentum. Usually additional fermion states would appear, hampering a lattice model by overloading its phenomenologic content ("species doubling"). The assumptions of the nogo theorem are:

(i) a local homogeneous (translation invariant on the lattice) hermitian hamiltonian, bilinear in the fermion fields;

(ii) locally defined, conserved and quantized charges bilinear in the fermion fields (such as the chiral charge).

The dilemma established by the no-go theorem is as follows: there can be no local, unitary, charge conserving theory on the lattice without equal numbers of left- and right-handed fermions for each combination of quantum numbers (charges). But the phenomenology we perceive in nature (for instance in the electroweak interactions the hypercharges $\left.2 Y\left(v_{\mathrm{L}}\right)=2 Y\left(\mathrm{e}_{\mathrm{L}}\right)=Y\left(\mathrm{e}_{\mathrm{R}}\right)=1\right)$ conserves charges, is unitary and has no extra fermions ( such as $\left.2 Y\left(v_{R}\right)=2 Y\left(e_{R}\right)=Y\left(e_{L}\right)=1\right)$ emerging from putting the field on the lattice [7]. Hence, lattice models and therefore also cellular automata concepts fail in this context.

5. One obvious way out of this dilemma is to give up locality of the transition law (2). In what follows a promising lattice fermion model is discussed, satisfying all other conditions despite locality [10-13]. The basic technical ingredient is the application of the finite element method to quantum field theory. There, undifferentiated fields appear as averages and derivatives as forward differences on the lattice. As a result the free Dirac equation (i $\gamma \partial-m) \psi(x)=0$ becomes [13] (Planck's constant $h=1$ and the velocity of light $c=1$, if not denoted otherwise):

$$
\left(\mathrm{i} \sum_{\mu=0}^{3} \gamma^{\mu} \sum_{\{\epsilon\}} \frac{(-1)^{\epsilon_{\mu}}}{a(\mu)}-\frac{1}{2} m \sum_{\{\epsilon\}}\right) \psi(n-\epsilon)=0 .
$$

Here, $a(\mu)$ is the lattice parameter in the $\mu$ direction, $\epsilon_{\mu}$ is 0 or 1 for each $\mu=0, \ldots, 3$, and $(n-\epsilon)$ stands for $\left(n_{0}-\epsilon_{0}, \ldots, n_{3}-\epsilon_{3}\right), n_{\mu} \in \mathbb{N}$. With the help of the Fourier transformation

$$
\psi(n)=\frac{1}{(2 \pi)^{4}} \int_{-\pi}^{\pi} \mathrm{d} \omega \int_{-\pi}^{\pi} \mathrm{d} p \tilde{\psi}(E, p) \exp \left[\mathrm{i}\left(a_{0} n_{0} E-a_{n} \cdot \boldsymbol{p}\right)\right],
$$

where $a(j) \approx$ const. $=a$ has been assumed, the following dispersion relation is obtained:

$$
\tan ^{2}\left(E a_{0} / 2\right)=\sum_{j=1}^{3}\left(a_{0} / a\right)^{2} \tan ^{2}\left(p_{j} a / 2\right)+\left(m a_{0} / 2\right)^{2},
$$

which in the limit $E, p_{j} \ll 1$ reduces to

$$
E^{2}=\boldsymbol{p}^{2}+m^{2} \text {. }
$$


Furthermore, it can be shown that the equal-time anticommutator relations are satisfied [13]:

$$
\left\{\psi^{\dagger}\left(n_{0}, \boldsymbol{n}\right), \psi\left(n_{0}, \boldsymbol{m}\right)\right\}=\delta_{n, m} /(2 \pi)^{3} .
$$

Given all field values $\psi\left(n_{0}-1, \boldsymbol{n}\right)$ at a time $n_{0}-1$, and the spatial Fourier transform $\tilde{\psi}\left(n_{0}-1, \boldsymbol{p}\right), \psi(n)$ at the next time step $n_{0}$ is explicitly given by

$$
\psi(n)=\frac{1}{(2 \pi)^{3}} \int_{-\pi}^{\pi} \tilde{D}(p) \tilde{S}(p) \mathrm{e}^{-\mathrm{i} p \cdot n} \mathrm{~d} p,
$$

where, after some calculation [13],

$$
\begin{aligned}
& \tilde{D}(p)=-\frac{\mathrm{i} \gamma_{0} / a_{0}+\sum_{j=1}^{3}\left(\gamma_{j} / a\right)^{-1} \tan \left(p_{i} / 2\right)+1 m / 2}{\sum_{\{\epsilon\}} \mathrm{e}^{\mathrm{i} p \cdot \varepsilon}\left[a_{0}^{-2}+\sum_{j=1}^{3} a^{-2} \tan ^{2}\left(p_{j} / 2\right)+(m / 2)^{2}\right]}, \\
& \tilde{D}(p)=\left(\mathrm{i} \gamma_{0} \sum_{\{\epsilon\}} \frac{\mathrm{e}^{\mathrm{i} p \cdot s}}{a_{0}}-\mathrm{i} \sum_{j=1}^{3} \gamma_{j} \sum_{\{\epsilon\}} \frac{(-1)^{\epsilon_{j}}}{a} \mathrm{e}^{\mathrm{i} p \cdot s}+1 \frac{m}{2} \sum_{\{\epsilon\}} \mathrm{e}^{\mathrm{i} p \cdot s}\right) \tilde{\psi}\left(n_{0}-1, p\right) .
\end{aligned}
$$

This transition law is nonlocal, since for the determination of $\tilde{\psi}$ knowledge about all cells $\psi\left(n_{0}-1, n\right)$ is necessary. Furthermore, the complex field amplitudes $\tilde{\psi}$ in (8) are no discrete state functions. Since locality and discrete states are indispensable criteria for cellular automata, the above model resembles more an array of (fictive) real number (not only floating point) processor elements with infinite storage capacity, each cell connected to any other cell of the array.

6. In what follows, a discretisation of the field amplitude $\psi$ is proposed, such that the energy and momentum variables take on discrete values per site $(n, n)$, in particular

$$
E=k_{0} e_{0}, \quad p_{j}=k_{j} p,
$$

where $k_{0}, k_{j} \in \mathbb{N}$ and $e_{0}, p \in \mathbb{R}$. Furthermore, a quantum condition on the product of canonical conjugate parameter pairs $\left(a_{0}, e_{0}\right)$ and $(a, p)$ could be imposed,

$$
a_{0} e_{0}=a p=1 \text {, }
$$

where unity stands for Planck's constant. The discrete field states can then be defined by the state function $\psi\left(n_{0}, \boldsymbol{n}, k_{0}, \boldsymbol{k}\right)$, very much resembling the Wigner function. By defining $\mu:=2 \tan ^{-1}(m / 2)$ dispersion relation (5) for some motion along a $p_{j}$-axis reads

$$
\tan ^{2}\left(k_{0} / 2\right)=\tan ^{2}\left(k_{j} / 2\right)+\tan ^{2}(\mu / 2) .
$$

For the low-momentum regime $k_{j}=0, k_{0}\left(k_{j}=0, \mu\right)=\mu$, whereas for the "massless" case $\mu=0, k_{0}\left(k_{j}, \mu=0\right)=k_{j}$. However, a physical interpretation of this procedure remains to be given.

7. Another way out of the dilemma established by the no-go theorem may be the abandonment of (locally defined) point particles and their associated quantum numbers (charges etc.). For extended "smeared out" charges [5] the no-go theorem is inapplicable, and fermion doubling could be avoided [14].

8. A third possibility to circumvent the no-go theorem may be a higher-dimensional configuration space with dimension $D>4$. In this case, it has to be assumed that for some (yet unknown) reason, dimensional reduction to $D=4$ occurs, such that the four-dimensional phenomenology is a "shadow" of a higher-dimensional world. I shall refer to this as "dimensional shadowing".

The projection of a higher-dimensional local lattice (with nearest neighbor connections) onto a lower-dimen- 
sional space, yields nonlocal connections (topologies) in the latter one, see fig. 2. More precisely, when there exists a density $n_{i}\left(V_{i}(D)\right)$ of point particles per $D$-volume element $V_{i}(D)$ and a finite overall number of particles $N=\sum_{i} n_{i}\left(V_{i}(D)\right)=$ const., then $\lim _{D \rightarrow \infty}\left\{n_{i}\left(V_{i}\right)-\delta_{i}^{(D)}\right\} \approx 0$. In particular, any finite number $N$ of points can be rearranged such that there exists a "critical dimension" $D^{*}=N-1$ associated with an $N$-simplex for which there is exactly one point per $D^{*}$-dimensional volume element and all points are locally connected. Projection of this $D^{*}$-dimensional lattice onto any hyperspace with $D<D^{*}$ yields nonlocal connectivities. Hence, in order to be able to reproduce connectivity of every cell with all other cells of the infinite array of (3)-(8) by dimensional shadowing, $D^{*}=\infty$.

9. It has been shown that putting a local fermion field theory on a tesselated space equivalent to its dual lattice yields well-known problems of species doubling. There may be several approaches to circumvent these obstruction. Three of those have been discussed. (i) Giving up the locality of the transition law. This has been discussed in the framework of recent developments in the theory of finite elements. The approach would get support from the nonlocality assumption in the epistomologic interpretation of the EPR paradoxon. (ii) Giving up local definition of quantum numbers which appear as "smeared out" charge sources etc. This method is very similar to saying that a particle is a composit of several cells. (iii) Dimensional shadowing from a high-dimensional (presumedly $\infty$-dimensional) space onto four-dimensional configuration space.

The topological equivalence between a tesselated space and cellular automata on the one hand and lattice field theory on the other hand allows one to apply theorems established in either area of research to the other one. For instance, it immediately follows that a local lattice field theory is a universal computer, a result established for continuum field theory recently [15]. Moreover, local lattice field theory is able to construct any embeddable field configuration (in particular replicas), and then set this configuration free. Whether such a field can produce configurations more sophisticated than itself out of its own and with no specification from the outside seems to be an unanswered question at present.

The author acknowledges discussions with Anton Zeilinger. This work was supported by BMWF, project number $19.153 / 3-26 / 85$.

\section{References}

[1] St. Wolfram, Rev. Mod. Phys. 55 (1983) 601.

[2] G.Y. Vichniac, Physica D 10 (1984) 96

[3] T. Toffoli, Physica D 10 (1984) 117.

[4] N. Margolus, Physica D 10 (1984) 81.

[5] M. Minsky, Int. J. Theor. Phys. 21 (1982) 537.

[6] R.P. Feynman, Int. J. Theor. Phys. 21 (1982) 467.

[7] H.B. Nielsen and M. Ninomiya, Nucl. Phys. B 185 (1981) 20; 193 (1981) 173.

[8] L.H. Karsten and J. Smith, Nucl. Phys. B 183 (1981) 103.

[9] J.M. Rabin, Phys. Rev. D 24 (1982) 468.

[10] R. Stacey, Phys. Rev. D 26 (1982) 468.

[11] C.M. Bender and D.H. Sharp, Phys. Rev. Lett. 50 (1983) 1535.

[12] C.M. Bender, K.A. Milton and D.H. Sharp, Phys. Rev. Lett. 51 (1983) 1815; Phys. Rev. D 31 (1985) 383.

[13] T. Matsuyama, Phys. Lett. B 158 (1985) 255.

[14] S.D. Drell, M. Weinstein and Y. Yankielowicz, Phys. Rev. D 14 (1976) 487.

[15] C.H. Woo, Phys. Lett. B 168 (1986) 376. 